\title{
SITUAÇÃO EPIDEMIOLÓGICA DA COQUELUCHE EM SALVADOR - BAHIA NOS ANOS DE 2012 A 2014
}

\author{
Ana Maria Cruz Santos*, Kelly Cerqueira Medeiros** \\ Autor correspondente: Kelly Cerqueira Medeiros kel.cm@hotmail.com \\ * Enfermeira; Especialista em Saúde da Criança e do Adolescente; Professora Auxiliar do Curso de Enfermagem da Escola \\ Bahiana de Medicina e Saúde Pública. Salvador-Bahia, Brasil., \\ ** Enfermeira, graduada pela Escola Bahiana de Medicina e Saúde Pública. Salvador-Bahia, Brasil.
}

\begin{abstract}
Resumo
O presente estudo objetivou descrever a situação epidemiológica da coqueluche no município de Salvador no estado da Bahia nos anos de 2012 a 2014. Trata-se de um estudo descritivo com abordagem quantitativa através da utilização de dados secundários disponíveis no Sistema de Informação de Agravos de Notificação do Ministério da Saúde. Os resultados apontam uma evidência elevada de notificações na primeira infância, com maior incidência nos primeiros meses de vida. Frente a esse cenário, o Ministério da Saúde ampliou o público-alvo das campanhas de vacinação, incluindo a vacina tríplice acelular para gestantes, visando garantir proteção aos recém-nascidos até que completem o esquema básico de vacinação. Conclui-se que, além do reforço da manutenção de cobertura vacinal, é de suma importância a inclusão da coqueluche nos diagnósticos diferenciais de tosse prolongada, provendo tratamento precoce aos doentes e permitindo a adoção de medidas de controle capazes de prevenir a expansão da doença a indivíduos suscetíveis.
\end{abstract}

Palavras-chave: Coqueluche; Imunização; Vigilância Epidemiológica.

\section{EPIDEMIOLOGICAL SITUATION OF PERTUSSIS IN SALVADOR - BAHIA IN YEAR 20122014}

\begin{abstract}
This study aimed to describe the epidemiological situation of pertussis in the city of Salvador, Bahia in the years 2012 to 2014. This is a descriptive study with a quantitative approach through the use of available secondary data in the Information system notification the Ministry of Health. The results show high evidence notifications in early childhood, focusing the first months of life. Faced with this scenario, the Ministry of Health expanded the target audience of vaccination campaigns, including the triple acellular vaccine for pregnant women in order to ensure protection of newborns until they

- Artigo submetido para avaliação em 26/10/2015 e aceito para publicação em 01/12/2015 •
\end{abstract}


complete the basic vaccination. We conclude that in addition to strengthening the immunization coverage of maintenance, it's very important to include whooping cough in the differential diagnosis of prolonged cough, providing early treatment to patients and allowing the adoption of control measures to prevent the spread of disease susceptible individuals.

Keywords: Pertussis; Immunization; Epidemiological Surveillance.

\section{INTRODUÇÃO}

Define-se coqueluche como doença infecciosa, aguda, transmissível e de distribuição universal comprometendo especificamente o aparelho respiratório. Distinguida, sobretudo, por paroxismos de tosse seca, (1) a bactéria Bodertella pertussis é eliminada através de gotículas de secreção da orofaringe, durante a fala, tosse e espirro², meio pelo qual advém a transmissão da doença. No Brasil, é considerada como agravo de notificação compulsória e constitui um grave problema de Saúde Pública. ${ }^{(1,3)}$

Por ano, são registrados 30 a 50 milhões de casos no mundo e 300 mil mortes, acometendo, em maior magnitude, os menores de seis meses, ${ }^{(5)}$ e, em lactentes, pode resultar em evoluções graves e até óbito. ${ }^{1,3)}$ Entre as principais complicações, estão a pneumonia, otite média, ativação de tuberculose latente, enfisema pneumotórax. ${ }^{(4)} \mathrm{A}$ realização do diagnóstico ocorre na maioria dos casos na fase paroxística, quando sucede a manifestação característica da doença, como as crises de tosse que podem ser acompanhadas de vômitos, cianose e apneia que duram de uma a seis semanas. ${ }^{(1,3)}$

Tem sido observada, nos últimos anos, uma elevada incidência de casos confirmados do agravo no país ${ }^{(4.5)}$ e, notadamente no estado da Bahia, não apenas na faixa etária de maior vulnerabilidade, mas também em pré-escolares, adolescentes e adultos. ${ }^{(4)} A$ situação epidemiológica nacional não difere do que é apresentado nos relatórios de outros países em relação ao elevado registro de casos nos últimos anos. ${ }^{(6)}$
$\mathrm{Na}$ última década, a coqueluche passou a ser considerada como uma doença reemergente em países desenvolvidos, devido à ocorrência de casos em adolescentes e adultos. $O$ aumento da incidência de casos em faixas etárias diferentes das comumente acometidas pela doença tornou-se um obstáculo para o diagnóstico, sendo este feito de forma tardia, ou por vezes não sendo feito, aumentando o risco de disseminação da doença para crianças lactentes e pré-escolares não vacinados. ${ }^{(7)}$

Previne-se a coqueluche por meio do cumprimento do calendário vacinal com a pentavalente (DTP + $\mathrm{HiB}+$ Hepatite B) aos dois, quatro e seis meses de vida, mais o reforço com a DTP aos 15 meses e aos quatro anos, ambas disponíveis pelo Sistema Único de Saúde, na rede de atenção básica. ${ }^{(1,3)}$

Segundo a Organização Mundial de Saúde, a cobertura de pelo menos $90 \%$ de crianças com três doses da vacina de alta qualidade continua a ser prioridade do programa de imunização em todo mundo. Seu principal objetivo é reduzir o risco de coqueluche grave na infância ${ }^{(8)}$. É de suma importância o papel que as vacinas exercem na proteção da saúde e na prevenção de agravos, especialmente durante a infância, em função disso, ${ }^{(9)}$ o ministério da Saúde estabelece meta de $95 \%$ de cobertura vacinal, e mesmo tendo demonstrado índices satisfatórios, no nordeste brasileiro, tem permanecido abaixo do que é preconizado. ${ }^{(9,10)}$ Além disso, as altas coberturas vacinais, ao determinarem uma redução de casos, tendem a diminuir também o reforço da exposição natural à doença. ${ }^{(1)}$ 
Desse modo, este estudo tem por objetivo descrever a situação epidemiológica da coqueluche no município de Salvador no estado da Bahia, nos anos de 2012 a 2014.

\section{MATERIAIS E MÉTODOS}

Trata-se de um estudo descritivo com uma abordagem quantitativa. Método utilizado para descrever as particularidades de determinadas populações ou fenômenos. Uma de suas características está na utilização de técnicas padronizadas de coleta de dados, tais como: pesquisa referente à idade, sexo, procedência e eleição. ${ }^{(12)}$ Quanto aos aspectos éticos, não cabe submissão ao Comitê de Ética em Pesquisa devido a base de dados ser de acesso público, sem possiblidade de identificação de dados pessoais confidenciais. A população estudada compreende todos os casos notificados ou confirmados de coqueluche no município de Salvador no estado da Bahia, registrados no Sistema Nacional de Agravos de Notificação (SINAN) no período de janeiro de 2012 a dezembro de 2014. Os dados secundários utilizados referentes à ocorrência de casos de coqueluche foram colhidos na página eletrônica da base de dados do Departamento de Informática do SUS (DATASUS). Os dados populacionais foram pesquisados na página eletrônica do Instituto Brasileiro de Geografia e Estatística (IBGE). A ferramenta utilizada para a análise da situação epidemiológica da coqueluche no período em estudo foi a construção de gráficos no Microsoft Excel 2010 compostos pelo total de casos notificados ou confirmados de acordo com as seguintes variáveis: período, sexo, faixa etária e óbitos. Para tal, foi empregado o cálculo de taxa e percentual de casos por período num histórico de três anos, de 2012 a 2014, por representarem a condição epidemiológica mais recente. Após a alimentação dos dados, foi possível descrever a situação da coqueluche nos últimos três anos no
Município de Salvador-BA, o que permitiu desenvolver uma análise crítica para a conclusão do presente estudo.

\section{RESULTADOS E DISCUSSÃO}

No Brasil eram notificados mais de 40 mil casos anuais no início da década de 80 , esse número caiu inesperadamente a partir de 1983, mantendose em uma tendência decrescente como resultado da cobertura vacinal.(5) No entanto, a partir de 2011, observou-se um aumento abrupto no número de casos no país, cuja incidência quadriplicou em relação à 2010. ${ }^{(13)}$

Nos três últimos anos no estado da Bahia, foram confirmados 972 casos de coqueluche, os dados do sistema de informação de agravos de notificação, revelam que o município mais afetado é o de Feira de Santana, correspondendo a 31,7\% dos casos do estado, totalizando 308 registros do agravo. Esses dados, quando comparados no mesmo período aos do município de Salvador o segundo com maior incidência $(23,3 \%)$ do agravo no estado revela uma elevação brusca no número de notificações.

Observa-se que esse registro se mantém numa tendência crescente (Gráfico 1), com o mínimo de 31 casos $(20,8 \%)$ registrados no ano de 2012 . Sustentando essa ascensão, as notificações chegam a duplicar em 2013 em relação ao ano anterior, totalizando um percentual de $21,6 \%$ o que corresponde a 68 casos confirmados de coqueluche. A taxa de incidência estimada em 2014 é de 4,4/100.000 habitantes, chegando a notificar 128 (25,5\%) ocorrências da doença no município de Salvador-BA, acometendo, em maior proporção, o sexo feminino, expresso no Gráfico 2. O aumento no número de casos no município em estudo e em todo o estado deve ser considerado, segundo a Secretaria de Saúde do Estado, uma vez que, pode estar associado com as novas recomendações sobre os critérios de suspeita, confirmação e tratamento dos casos. $(1,10,14)$ No estado de São Paulo, foi implantado no 
ano 2000 pela Divisão de Doenças de Transmissão Respiratória/CVE/CCD/SES-SP, um sistema de vigilância para coqueluche com base em unidades sentinelas, com o objetivo de triar os casos desde a suspeita, confirmação diagnóstica, por meio da coleta de secreção da nasofaringe, até o acompanhamento da tendência da doença. ${ }^{(15)}$

Gráfico1 - Casos confirmados de coqueluche. Salvador e Bahia 2012 a 2014

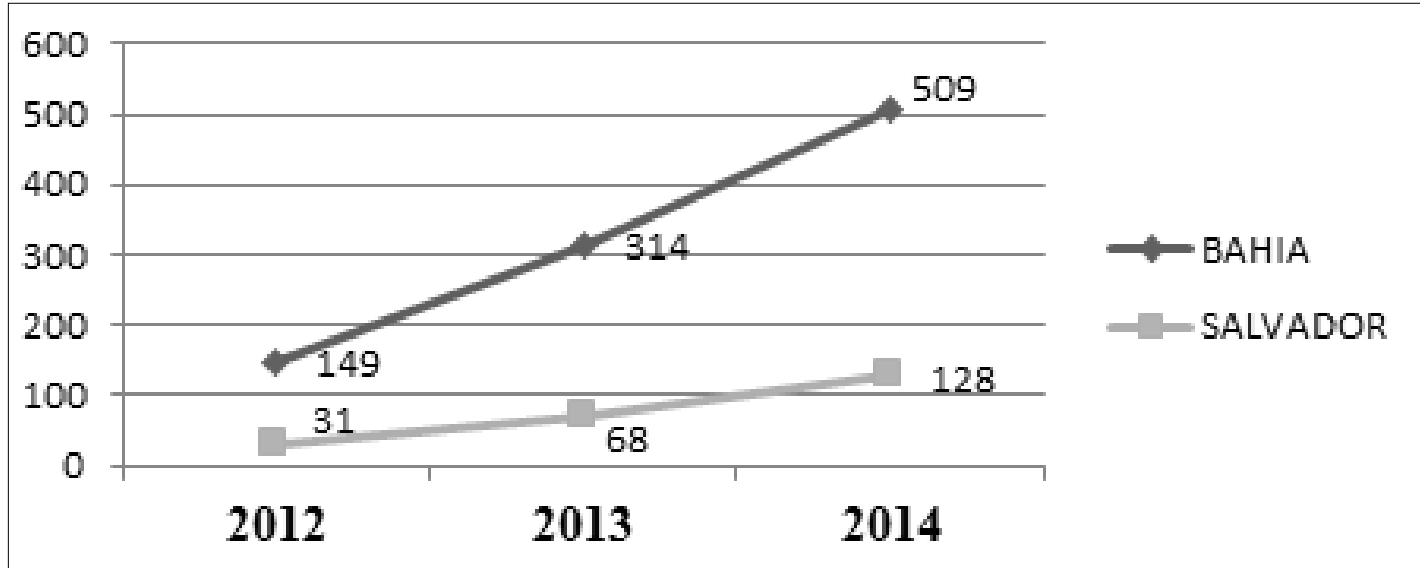

Fonte: Elaborado pelas autoras com base nos dados coletados no Sistema Nacional de Agravos de Notificação (SINAN).

Gráfico 2 - Casos confirmados de coqueluche por sexo. Salvador - Bahia, 2012 a 2014

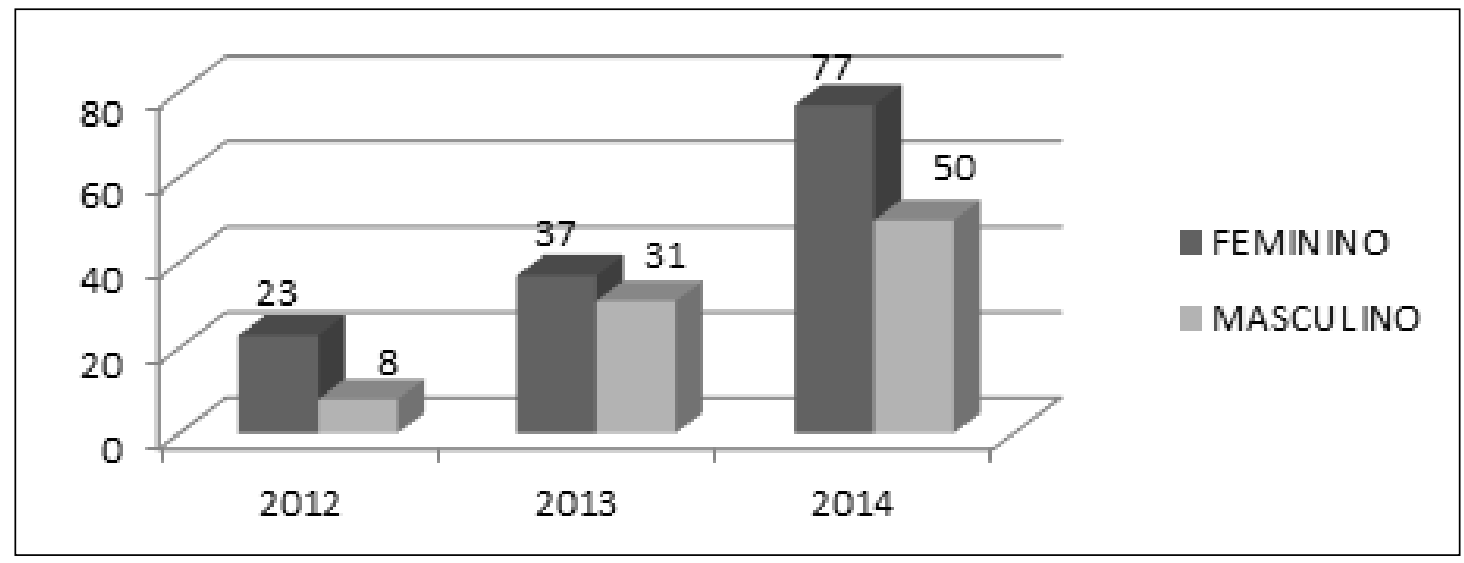

Fonte: Elaborado pelas autoras com base nos dados coletados no Sistema Nacional de Agravos de Notificação (SINAN).

Embora nos últimos anos a incidência da mortalidade em menores de um ano tenha reduzido em relação aos anos anteriores, sucedida do impacto positivo da imunização na primeira infância, a coqueluche continua a ser uma preocupação de saúde pública, devido à elevação no número de casos. ${ }^{(16)} \mathrm{Na}$ Bahia foram registrados sete óbitos no período em estudo, dois deles incidiram no município de Salvador, informados em 2013 e 2014 respectivamente. Ambos ocorreram em menores de seis meses de idade (Gráfico 3), grupo que em função da faixa etária, possui maior propensão a desenvolver as formas graves da doença por não apresentar esquema vacinal básico. ${ }^{(2,16)}$ 
Gráfico 3 - Casos confirmados e óbitos em menores de um ano por coqueluche. Salvador - Bahia, 2012 a 2014

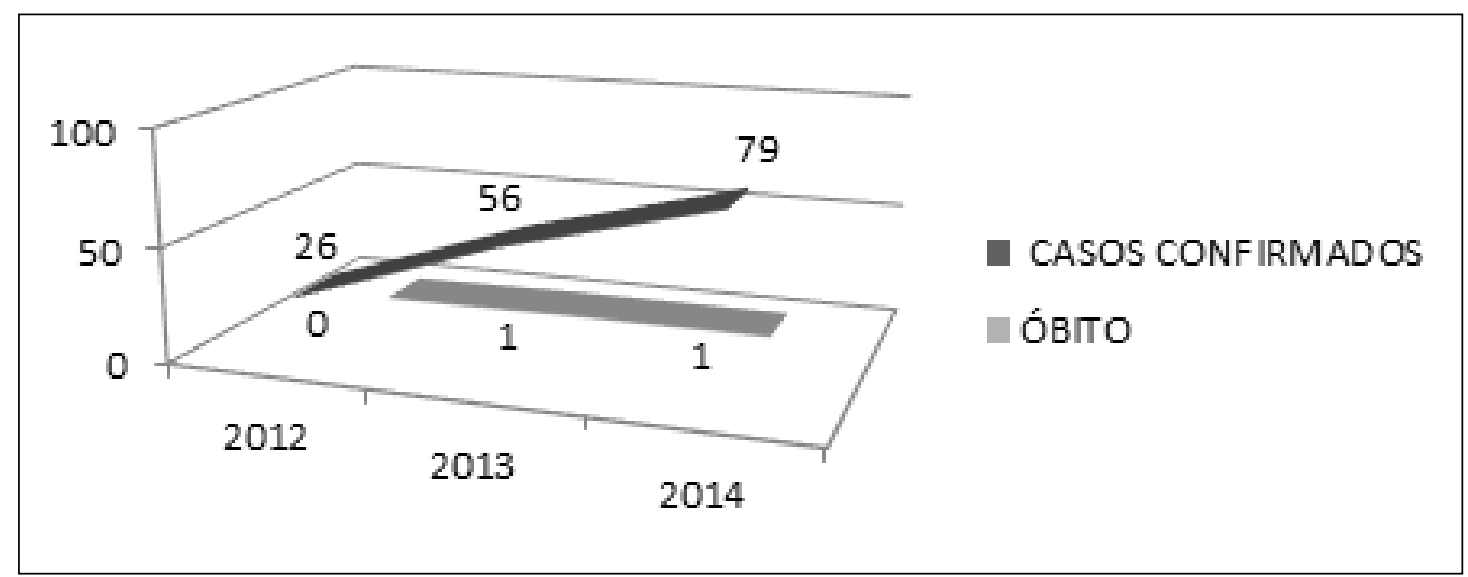

Fonte: Elaborado pelas autoras com base nos dados coletados no Sistema Nacional de Agravos de Notificação (SINAN).

Analisando a notificação da doença no município de Salvador-BA por faixa etária, observa-se a ocorrência em todas as idades. A imunidade adquirida artificialmente não é permanente e perde sua eficácia entre cinco a dez anos após a ultima dose da vacina, ${ }^{(1,3,5)}$ dessa forma, a coqueluche, além de atingir as crianças com esquema vacinal incompleto, afeta também as crianças vacinadas maiores de quatro anos de idade, adolescentes e principalmente os adultos com idade entre 20 a 39 anos, equivalendo a 7,9\% dos registros. Nos países desenvolvidos o índice de casos também aumentou consideravelmente, e apesar das coberturas vacinais, a doença tem reaparecido em todas as idades, o que ocasionou a indicação de um reforço em adolescentes e adultos. ${ }^{(15)}$
Em relação à distribuição desses casos, o grupo etário mais atingido é o de menores de um ano com 161 casos confirmados, correspondendo a $70,9 \%$ das notificações nos três últimos anos, visto que se trata da faixa etária de maior vulnerabilidade. Esses percentuais também demonstram a proporção de $11 \%$ da ocorrência da doença entre crianças de 01 a 04 anos (Gráfico 4). Frente a essa situação, o Ministério da Saúde ampliou o público-alvo das campanhas de vacinação e, a partir do segundo semestre de 2014, disponibilizou a vacina tríplice acelular (DTPa) para gestantes com o intuito de garantir proteção aos recém-nascidos até que completem o esquema básico de vacinação com a pentavalente, aos seis meses de vida. ${ }^{(4)}$

Gráfico 4 - Casos confirmados de coqueluche por faixa etária em Salvador - Bahia. 2012 a 2014.

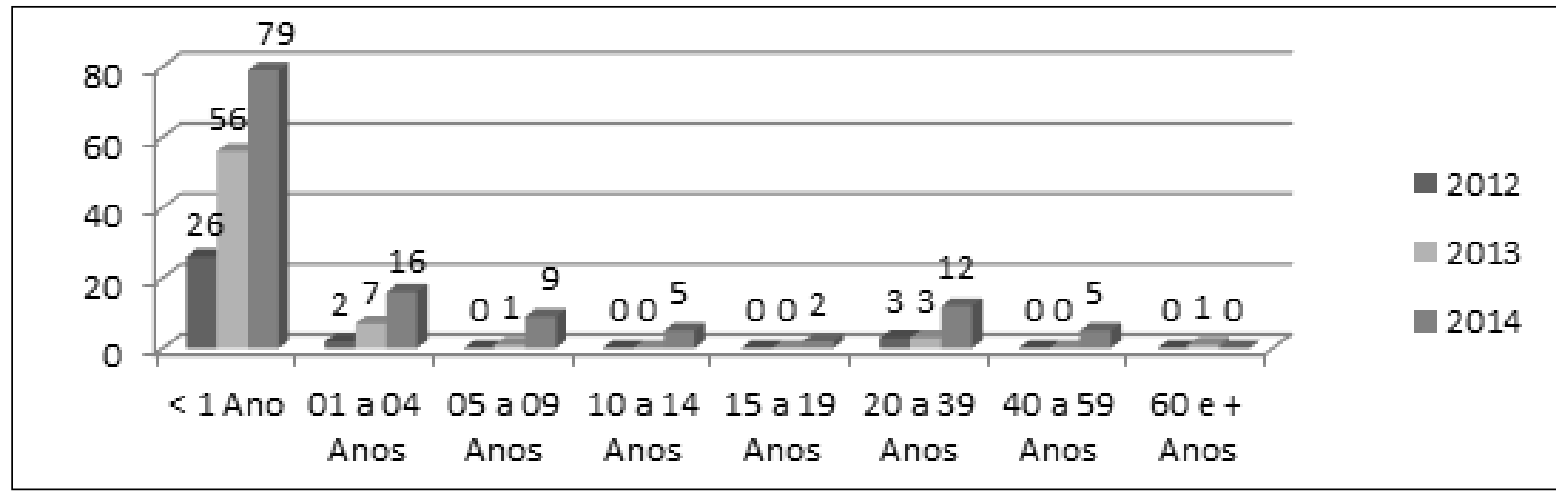

Fonte: Elaborado pelas autoras com base nos dados coletados no Sistema Nacional de Agravos de Notificação (SINAN). 


\section{CONCLUSÃO}

Com esse estudo foi possível caracterizar a situação epidemiológica no município de Salvador no estado da Bahia, o segundo com maior número de registros no SINAN, onde se constatou que a coqueluche representa um grave problema na saúde da população, e que mesmo com a disponibilidade de vacinas eficazes e altas coberturas vacinais, continua ocorrendo de maneira crescente, acometendo adultos, crianças e especialmente os lactentes. O que determina a expansão da doença nos diferentes grupos etários é o fator imunidade. Tanto a exposição ao agente, quanto a imunização, não conferem defesa permanente ao indivíduo exposto, contribuindo diretamente na extensão da doença a indivíduos vulneráveis.

Nesse sentido, deve-se reforçar a inclusão coqueluche nos diagnósticos diferenciais de tosse prolongada pelos profissionais de saúde, uma vez que, provê tratamento precoce aos doentes, favorece a adoção de medidas de controle capazes de prevenir a expansão da coqueluche a indivíduos suscetíveis e o acompanhamento dos resultados das medidas que vêm sendo executadas. Além disso, é possível determinar os fatores envolvidos no aumento do número de casos gerando dados para elaboração de novos estudos e promovendo a implementação de medidas mais específicas para combater o desenvolvimento da doença.

\section{REFERÊNCIAS}

1. Bahia. Secretaria de Saúde do Estado da Bahia. Diretoria de Vigilância Epidemiológica. Protocolo de vigilância epidemiológica da coqueluche na Bahia. Salvador; 2013.

2. Brasil. Secretaria de Vigilância em Saúde. Departamento de Vigilância Epidemiológica. Doenças infecciosas e parasitárias: guia de bolso. Brasília, DF; 2010.

3. Brasil. Secretaria de Vigilância em Saúde. Guia de vigilância em Ssúde. Brasília, DF; 2014. [internet]. Disponível em: http://portalsaude. saude.gov.br/ Acesso em: 21 mar. 2015.

4. Brasil. Secretaria de Vigilância em Saúde. Alerta sobre a situação epidemiológica da coqueluche no Brasil. Brasília, DF; 2O13. [internet].

Disponível em: http://www.portalsaude.saude. gov.br/bahia-deve-vacinar-210-mil-gestantescontra coqueluche. Acesso em: 21 mar. 2015.

5. Brasil. Guia de vigilância epidemiológica. $7^{\mathrm{a}} \mathrm{ed}$. Brasília, DF; 2009. [internet]. Disponível em: www.saude.gov.br/svs Acesso em: O2 mar. 2015.

6. Trevisan S, Coutinho SED. Perfil Epidemiológico da coqueluche no Rio Grande do Sul, Brasil: estudo da correlação entre incidência e cobertura vacinal. Cad. saúde pública. [periódico na internet]. 2008 [acesso em 27 set. 2015];24(1). Disponível em: http://www. saude.sc.gov.br/

7. Ceará. Secretaria da Saúde do Ceará. Alerta para notificação de casos suspeitos de Coqueluche no Ceará. Ceará; 2013. [internet]. Disponível em: http://www.saude.ce.gov.br Acesso em: O2 mar. 2015.

8. Organização Mundial de Saúde. Pertussis. Imunization, Vaccines and Biologicals. 2015. [internet]. Disponivel em: http://www.who.int/ topics/pertussis/en/index.html Acesso em: $\mathrm{O} 2$ mar. 2015.

9. Queiroz LLC, Monteriro SG, Mochel EG, Veras MASM, Sousa FGM, Bezerra MLM et al. Cobertura vacinal do esquema básico para o primeiro ano de vida nas capitais do Nordeste brasileiro. Cad. saúde pública. 2013;29(2):294$3 \mathrm{O} 2$.

10. Bahia. Secretaria de Saúde da Bahia. Implantação e implementação das campanhas com as vacinas: Tríplice Viral (SRC) e a Poliomielite 1, 2 E 3 (Atenuada) com ênfase na introdução da vacina Adsorvida Difteria, Tétano e Pertussis (Acelular) dTpa, adulto com a Divulgação da Portaria GM/MS nº 1.498 de 19/O7/2O13. Salvador: Sesab; 2O14. [internet]. Disponível em: www.suvisa.ba.gov.br Acesso em: O2 out. 2015.

11. Cherry JD. Epidemic Pertussis in 2012: the ressurgence of a vaccine-preventable disease. $\mathrm{N}$. Engl. j. med. [periódico na internet] $2 \mathrm{Ol} 2$ [acesso em 05 out. 2015];367(9):785-787. Disponível 
em: http://www.nejm.org/doi/full/10.1056/

NEJMp1209051"\#sthash.rs5hCvoD.dpuf

12. Gil AC. Como elaborar projetos de pesquisa. $4^{\mathrm{a}}$ ed. São Paulo: Atlas; 2008.

13. Pernambuco. Secretaria Estadual de Saúde. Retificação da Nota Informativa nO 8/2O14 sobre as novas recomendações para vigilância epidemiológica da coqueluche. Recife; 2014. Disponível em: http://portal.saude.pe.gov.br/ Acesso em: $\mathrm{O} 6$ out. 2015.

14. Bahia. Diretoria de Vigilância Epidemiológica. Relatório de Gestão 2014 (SE 4O). Salvador: Sesab; 2014. Disponível em: http://portalsaude. saude.gov.br/index.php/o-ministerio/principal/arquivos Acesso em: $\mathrm{O} 2$ out. $2 \mathrm{Ol5}$.

15. São Paulo (Estado). Secretaria de Estado da Saúde de São Paulo. Divisão de Doenças de
Transmissão Respiratória. Centro de Vigilância Epidemiológica "Prof. Alexandre Vranjac". Coordenadoria de Controle de Doenças. Situação epidemiológica atual da coqueluche Cenário global. BEPA; 2012;9(97):26-35.

16. Korppi M. Whooping cough: still a challenge. J. pediatr. 2013;89(6):520-522.

17. Brasil. Sistema Nacional de Agravos de Notificação. Disponível em: http://dtr2004. saude.gov.br/sinanweb/index.php Acesso em: 14 jan. 2015.

18. Brasil. Departamento de Informática do SUS. Disponível em: http://www.datasus.gov.br Acesso em: 14 jan. 2015.

19. Instituto Brasileiro de Geografia e Estatística. Disponível em: http://www.ibge.gov.br/home Acesso em: 15 jan. 2015. 